\title{
ON WEIGHTED INEQUALITIES FOR SINGULAR INTEGRALS
}

\author{
H. AIMAR, L. FORZANI, AND F. J. MARTÍN-REYES
}

(Communicated by J. Marshall Ash)

\begin{abstract}
In this note we consider singular integrals associated to CalderónZygmund kernels. We prove that if the kernel is supported in $(0, \infty)$ then the one-sided $A_{p}$ condition, $A_{p}^{-}$, is a sufficient condition for the singular integral to be bounded in $L^{p}(w), 1<p<\infty$, or from $L^{1}(w d x)$ into weak- $L^{1}(w d x)$ if $p=1$. This one-sided $A_{p}$ condition becomes also necessary when we require the uniform boundedness of the singular integrals associated to the dilations of a kernel which is not identically zero in $(0, \infty)$. The two-sided version of this result is also obtained: Muckenhoupt's $A_{p}$ condition is necessary for the uniform boundedness of the singular integrals associated to the dilations of a general Calderón-Zygmund kernel which is not the function zero either in $(-\infty, 0)$ or in $(0, \infty)$.
\end{abstract}

\section{INTRODUCTION}

It is a classical result in the theory of weighted inequalities the fact that the $A_{p}$ condition of B. Muckenhoupt on a weight $w$ is equivalent to the $L^{p}(w d x)$ boundedness of the Hilbert transform. This result was proved in 1973 by Hunt, Muckenhoupt and Wheeden [HMW]. In 1974 Coifman and Fefferman $[\mathrm{CF}]$ gave a different proof which relies on a good- $\lambda$ inequality, producing an integral estimate of the singular integral in terms of the Hardy-Littlewood maximal operator.

Since 1986 the work by E. Sawyer [S], Andersen and Sawyer [AS], Martín Reyes, Ortega Salvador and de la Torre $[\mathrm{MOT}],[\mathrm{MT}]$ has shown that many positive operators of real analysis have one-sided versions for which the classes of weights are larger than Muckenhoupt's ones. Our purpose here is to study the corresponding problems for singular integrals.

The situation for one-sided singular integrals is different. The symmetry properties of the Hilbert kernel produce the necessary cancellation properties of a singular integral, so that, no one-sided truncation of $1 / x$ is expected to produce a one-sided singular integral. Nevertheless, as we show in Lemma (1.5), the class of general singular integral Calderón-Zygmund kernels supported on a half line is nontrivial. We ask for the more general class of weights $w$ for which such singular integral operators are bounded in $L^{p}(w d x)$. It turns out (Theorem (2.1)) that the one-sided $A_{p}$ condition is a sufficient condition which becomes also necessary when we require

Received by the editors March 15, 1995 and, in revised form, January 30, 1996.

1991 Mathematics Subject Classification. Primary 42B25.

Key words and phrases. Singular integrals, Calderón-Zygmund operators, weights.

The research of the third author has been partially supported by D.G.I.C.Y.T. grant (PB910413) and Junta de Andalucía. 
the uniform boundedness of the singular integrals associated to the dilations of a kernel which is not the function zero in $(0, \infty)$ or in $(-\infty, 0)$ (Theorem $(2.6)$ ). The two-sided version of this result gives (see also Theorem (2.6)) that Muckenhoupt's $A_{p}$ condition is necessary for the uniform boundedness of the singular integrals associated to the dilations of a Calderón-Zygmund kernel which is not the function zero either in $(-\infty, 0)$ or in $(0, \infty)$.

\section{One-Sided Singular InTEGRALS}

We shall say that a function $k$ in $L_{l o c}^{1}(\mathbb{R}-\{0\})$ is a Calderón-Zygmund kernel if the following properties are satisfied:

(1.1) there exists a finite constant $B_{1}$ such that

$$
\left|\int_{\varepsilon<|x|<N} k(x) d x\right| \leq B_{1} \quad \text { for all } \varepsilon \text { and all } N \text {, with } 0<\varepsilon<N,
$$

and furthermore $\lim _{\varepsilon \rightarrow 0^{+}} \int_{\varepsilon<|x|<1} k(x) d x$ exists,

(1.2) there exists a finite constant $B_{2}$ such that

$$
|k(x)| \leq \frac{B_{2}}{|x|}, \quad \text { for all } x \neq 0,
$$

(1.3) there exists a finite constant $B_{3}$ such that

$$
|k(x-y)-k(x)| \leq B_{3}|y||x|^{-2} \text { for all } x \text { and } y \text { with }|x|>2|y|>0 .
$$

These conditions are known to be sufficient for the $L^{p}$ boundedness and the weak type $(1,1)$ of the maximal operator

$$
T^{*} f(x)=\sup _{\varepsilon>0}\left|T_{\varepsilon} f(x)\right|,
$$

with

$$
T_{\varepsilon} f(x)=\int_{|x-y|>\varepsilon} k(x-y) f(y) d y
$$

(see e.g. $[\mathrm{T}]$ ). In other words

$$
\left|\left\{x \in \mathbb{R}: T^{*} f(x)>\lambda\right\}\right| \leq \frac{C_{1}}{\lambda} \int_{\mathbb{R}}|f|
$$

and

$$
\left\|T^{*} f\right\|_{p} \leq C_{p}\|f\|_{p}
$$

for $1<p<\infty$, where $|E|$ stands for the Lebesgue measure of the set $E$ and $\|f\|_{p}=\left(\int|f(x)|^{p} d x\right)^{1 / p}$. The same inequalities hold for the singular integral

$$
T f(x)=\lim _{\varepsilon \rightarrow 0^{+}} T_{\varepsilon} f(x) .
$$

The next lemma shows that there exist nontrivial Calderón-Zygmund kernels with support contained in $(0, \infty)$.

(1.4) Lemma. The function

$$
k(x)=\frac{1}{x} \cdot \frac{\sin (\log x)}{\log x} \cdot \chi_{(0, \infty)}(x),
$$

is a Calderón-Zygmund kernel. 
Proof. To prove (1.1), take $0<\varepsilon<N<\infty$ and change variables in the integral $\int_{\varepsilon<|x|<N} k(x) d x$ to get

$$
\int_{\log \varepsilon}^{\log N} \frac{\sin t}{t} d t
$$

which is uniformly bounded in $\varepsilon$ and $N$ and, for fixed $N$, converges to a finite limit as $\varepsilon$ tends to zero. On the other hand, since the function $g(t)=t^{-1} \sin t$ is bounded by one, it is clear that $k$ satisfies (1.2) with $B_{2}=1$. Let us prove (1.3). Notice that the function $g(t)$ has a bounded derivative so that

$$
\left.\frac{d}{d x} k(x)=\frac{d}{d x} \frac{1}{x} g(\log x)\right\}=\frac{1}{x^{2}}\left\{g^{\prime}(\log x)-g(\log x)\right\},
$$

from which (1.3) follows with a constant $B_{3}$.

\section{Weighted INEQUALITIES}

The one-sided Hardy-Littlewood maximal functions are defined by

$$
M^{+} f(x)=\sup _{h>0} \frac{1}{h} \int_{x}^{x+h}|f| \text { and } M^{-} f(x)=\sup _{h>0} \frac{1}{h} \int_{x-h}^{x}|f| .
$$

It is a known result by E. Sawyer [S] that $M^{-}$applies $L^{p}(w d x)$ into $L^{p}(w d x)$, $1<p<\infty$, if and only if the weight $w$ satisfies

$$
A_{p}^{-}:\left(\int_{a}^{b} w^{-\frac{1}{p-1}}\right)^{p-1}\left(\int_{b}^{c} w\right) \leq C(c-a)^{p}
$$

for all numbers $a<b<c$ with a finite constant $C$ independent of $a, b$, and $c$.

Also $M^{-}$applies $L^{1}(w d x)$ into weak- $L^{1}(w d x)$ if and only if $w$ satisfies

$$
A_{1}^{-}: M^{+} w \leq C w \text { a.e. }
$$

Analogous results hold for the operator $M^{+}$and the corresponding $A_{p}^{+}$classes which are defined in the obvious way.

In the more recent paper [MPT] the one-sided analog of $A_{\infty}$ weights are studied. A weight $w$ is in $A_{\infty}^{-}$if and only if there exist positive numbers $C$ and $\delta$ such that for all numbers $a<b<c$ and all measurable sets $E \subset(a, b)$

$$
\frac{|E|}{c-a} \leq C\left(\frac{w(E)}{w(b, c)}\right)^{\delta}
$$

(as usual, $w(E)$ stands for $\int_{E} w(x) d x$ ). This class of weights is proved to coincide with the union of the $A_{p}^{-}$classes with $p \geq 1$. In fact, we have the following result:

Theorem A [MPT, Theorem 1]. Let $w$ be a weight, i.e., a positive, locally integrable function in the real line. The following are equivalent:

(a) There exists $p, 1 \leq p<\infty$, such that $w \in A_{p}^{-}$.

(b) $w$ is in $A_{\infty}^{-}$.

(c) There exist positive numbers $C$ and $\delta$ such that

$$
\frac{w(E)}{w(a, c)} \leq C\left(\frac{|E|}{b-a}\right)^{\delta}
$$

for all numbers $a<b<c$ and all measurable sets $E \subset(b, c)$. 
We are now in position to state the main results of this note.

(2.1) Theorem. Let $k$ be a Calderón-Zygmund kernel with support in $\mathbb{R}^{+}=(0, \infty)$. Then

(2.2) given a weight $w$ in $A_{\infty}^{-}$there exists a constant $C_{p}$ depending only on $B_{1}, B_{2}$, $B_{3}, p$ and the constants in the $A_{\infty}^{-}$condition, such that

$$
\int_{\mathbb{R}}\left|T^{*} f(x)\right|^{p} w(x) d x \leq C_{p} \int_{\mathbb{R}}\left|M^{-} f(x)\right|^{p} w(x) d x, \quad 1<p<\infty,
$$

and

$\sup _{\lambda>0} \lambda^{p} w\left(\left\{x: T^{*} f(x)>\lambda\right\}\right) \leq C_{p} \sup _{\lambda>0} \lambda^{p} w\left(\left\{x: M^{-} f(x)>\lambda\right\}\right), \quad 1 \leq p<\infty$,

for all $f \in L^{p}(w d x)$,

(2.3) given a weight $w \in A_{p}^{-}$with $1<p<\infty$ there exists a constant $C$ depending only on $B_{1}, B_{2}, B_{3}, p$ and the constant in the condition $A_{p}^{-}$, such that

$$
\int_{\mathbb{R}}\left|T^{*} f(x)\right|^{p} w(x) d x \leq C \int_{\mathbb{R}}|f(x)|^{p} w(x) d x,
$$

for all $f \in L^{p}(w d x)$,

(2.4) given a weight $w \in A_{1}^{-}$there exists a constant $C$ depending only on $B_{1}, B_{2}$, $B_{3}$ and the constant in the condition $A_{1}^{-}$such that

$$
w\left(\left\{x: T^{*} f(x)>\lambda\right\}\right) \leq \frac{C}{\lambda} \int_{\mathbb{R}}|f(x)| w(x) d x
$$

for all $f \in L^{1}(w d x)$ and all $\lambda>0$.

(2.5) Remarks. Let us remark here that similar right-hand-sided results hold with kernels supported on the negative real numbers, changing $M^{-}$to $M^{+}$and $A_{p}^{-}$to $A_{p}^{+}$.

Let $k$ be a Calderón-Zygmund kernel and let us consider for each $\alpha>0$ the dilation

$$
k_{\alpha}(x)=\alpha k(\alpha x) .
$$

It is clear that $k_{\alpha}$ is also a Calderón-Zygmund kernel with the same constants $B_{1}$, $B_{2}$ and $B_{3}$ as $k$. Therefore, Theorem (2.1) gives that the maximal singular integrals $T_{\alpha}^{*}$ associated to the dilations $k_{\alpha}$ are uniformly bounded in $L^{p}(w d x)$ if $w \in A_{p}^{-}$with $1<p<\infty$ and from $L^{1}(w d x)$ into weak- $L^{1}(w d x)$ if $w \in A_{1}^{-}$. The next theorem is a kind of converse of this remark.

(2.6) Theorem. Let $k$ be a Calderón-Zygmund kernel. For each $\alpha>0$, let $T_{\alpha}^{*}$ denote the maximal operator with kernel $k_{\alpha}(x)=\alpha k(\alpha x)$ and let $1 \leq p<\infty$. Let $w$ be a weight and assume that the operators $T_{\alpha}^{*}$ are uniformly bounded from $L^{p}(w d x)$ into weak- $L^{p}(w d x)$.

(a) If there exists $z>0$ such that $k(z) \neq 0$ then $w \in A_{p}^{-}$.

(b) If there exists $s<0$ such that $k(s) \neq 0$ then $w \in A_{p}^{+}$.

(c) If there exist $s<0<z$ such that $k(s) \neq 0 \neq k(z)$ then $w \in A_{p}$; i.e., $w$ belongs to the $A_{p}$ class of Muckenhoupt. 
Theorems (2.1) and (2.6) hold also for the singular integral

$$
T f(x)=\lim _{\varepsilon \rightarrow 0^{+}} T_{\varepsilon} f(x) .
$$

The proofs for $T$ are similar to the corresponding for $T^{*}$ or follow easily from the theorem for $T^{*}$.

Proof of Theorem (2.1). Theorem (2.1) is an easy consequence of Sawyer's results for $M^{-}[\mathrm{S}]$ and of the next lemma which is itself an extension, to the one-sided setting, of the good- $\lambda$ inequality of Coifman and Fefferman $[\mathrm{CF}]$.

(2.7) Lemma. Let $k$ be a Calderón-Zygmund kernel with support in $\mathbb{R}^{+}$. Let $w$ be a weight in $A_{\infty}^{-}$. Then there exist positive constants $C$ and $\gamma_{o}$ such that for every $0<\gamma \leq \gamma_{o}$ the inequality

(2.8) $w\left(\left\{x \in \mathbb{R}: T^{*} f(x)>2 \lambda, M^{-} f(x)<\gamma \lambda\right\}\right) \leq C \gamma^{\delta} w\left(\left\{x \in \mathbb{R}: T^{*} f(x)>\lambda\right\}\right)$,

$f \in L^{1}$ and every positive $\lambda$ with $\delta$ the exponent in statement (c) of Theorem A.

Proof of (2.7). Since the set $\left\{x: T^{*} f(x)>\lambda\right\}$ is open and has finite measure for $f$ in $L^{1}$, we have that it can be written as a disjoint countable union of open intervals. Let $J=(a, b)$ be such an interval. It is enough to prove that there exist $C$ and $\gamma_{0}$ such that

$$
w\left(\left\{x \in J: T^{*} f(x)>2 \lambda, M^{-} f(x)<\gamma \lambda\right\}\right) \leq C \gamma^{\delta} w(J),
$$

for every $0<\gamma \leq \gamma_{0}$ and every $\lambda>0$ (throughout the proof, $C$ will be a constant that may change from line to line.) Let us now take a sequence $\left\{x_{i}: i \in \mathbb{N}\right\}$ in $J=(a, b)$ in such a way that $x_{0}=b$ and $x_{i-1}-x_{i}=x_{i}-a$ for every $i \geq 1$. Since the weight $w \in A_{\infty}^{-}$we have by Theorem A (statement (c) that, in order to prove (2.9), we only need to show that

$$
\left|E_{i}\right| \leq C \gamma\left(x_{i+1}-x_{i+2}\right)
$$

where $E_{i}=\left\{x \in\left(x_{i+1}, x_{i}\right): T^{*} f(x)>2 \lambda, M^{-} f(x)<\gamma \lambda\right\}$. In fact, if (2.10) holds then by (c) in Theorem A we have that

$$
w\left(E_{i}\right) \leq C \gamma^{\delta} w\left(x_{i+2}, x_{i}\right) .
$$

Summing up in $i$ we obtain (2.9).

In order to prove (2.10) we fix $i \in \mathbb{N}$ and choose $\bar{a}<a$ in such a way that $x_{i}-a=a-\bar{a}$. Now decompose $f$ as $f_{1}+f_{2}$ with $f_{1}=f \chi_{(\bar{a}, \infty)}$. If $E_{i}=\emptyset$ there is nothing to prove. Let us write $\xi_{0}=\sup \left\{\xi \in\left(x_{i+1}, x_{i}\right): M^{-} f(\xi) \leq \gamma \lambda\right\}$ and notice that

$$
\begin{aligned}
E_{i} \subset\left\{x \in\left(x_{i+1}, \xi_{0}\right): T^{*} f_{1}(x)>\lambda / 2\right\} \\
\qquad\left\{x \in\left(x_{i+1}, x_{i}\right): T^{*} f_{2}(x)>\frac{3}{2} \lambda, M^{-} f(x) \leq \gamma \lambda\right\} .
\end{aligned}
$$

Since for $x \in\left(x_{i+1}, \xi_{0}\right), T^{*} f_{1}(x)=T^{*}\left(f \chi_{\left(\bar{a}, \xi_{0}\right)}\right)(x)$ because of the support property on $k$, applying the weak type $(1,1)$ of $T^{*}$ we have the desired bound for the measure of the first term on the right of (2.11):

$$
\begin{aligned}
\mid\left\{\left(x_{i+1}, \xi_{0}\right)\right. & \left.: T^{*} f_{1}>\lambda / 2\right\}\left|\leq \frac{C}{\lambda} \int_{\bar{a}}^{\xi_{0}}\right| f \mid \\
& \leq \frac{C}{\lambda}\left(\xi_{0}-\bar{a}\right) M^{-} f\left(\xi_{0}\right) \leq C \gamma\left(x_{i+1}-x_{i+2}\right) .
\end{aligned}
$$


We shall now prove that the second set on the right term of (2.11) is essentially empty for $\gamma$ small enough. Take $x \in\left(x_{i+1}, x_{i}\right)$ and $\varepsilon$ positive. Then, with $k^{(\varepsilon)}=$ $k \chi_{(\varepsilon, \infty)}$, we have

$$
\begin{aligned}
\left|T_{\varepsilon} f_{2}(x)\right| & =\left|\int_{-\infty}^{x} k^{(\varepsilon)}(x-y) f_{2}(y) d y\right|=\left|\int_{-\infty}^{\bar{a}} k^{(\varepsilon)}(x-y) f(y) d y\right| \\
& \leq\left|\int_{-\infty}^{\bar{a}}\left[k^{(\varepsilon)}(x-y)-k^{(\varepsilon)}(a-y)\right] f(y) d y\right|+\left|\int_{-\infty}^{\bar{a}} k^{(\varepsilon)}(a-y) f(y) d y\right| \\
& =\mathrm{I}+\mathrm{II} .
\end{aligned}
$$

Let us first estimate II. For $\varepsilon \geq a-\bar{a}$ we have

$$
\left|\int_{-\infty}^{\bar{a}} k^{(\varepsilon)}(a-y) f(y) d y\right|=\left|\int_{-\infty}^{a} k^{(\varepsilon)}(a-y) f(y) d y\right| \leq T^{*} f(a) \leq \lambda .
$$

If $\varepsilon<a-\bar{a}$ then

$$
\left|\int_{-\infty}^{\bar{a}} k^{(\varepsilon)}(a-y) f(y) d y\right|=\left|\int_{-\infty}^{a} k^{(a-\bar{a})}(a-y) f(y) d y\right| \leq T^{*} f(a) \leq \lambda .
$$

In order to finish the proof of the lemma, we only need to show that there exists $\gamma_{0}>0$ such that for every $0<\gamma \leq \gamma_{0}$ and $x \in\left(x_{i+1}, x_{i}\right)$ with $M^{-} f(x) \leq \gamma \lambda$ we have $\mathrm{I} \leq \lambda / 2$. Let $\chi_{\varepsilon}(t)$ denote the characteristic function of the half line $(\varepsilon, \infty)$. Then

$$
\begin{aligned}
\mathrm{I} & \leq \int_{-\infty}^{\bar{a}}\left|k(x-y) \chi_{\varepsilon}(x-y)-k(a-y) \chi_{\varepsilon}(a-y)\right||f(y)| d y \\
& \leq \int_{-\infty}^{\bar{a}}|k(x-y)-k(a-y)| \chi_{\varepsilon}(a-y)|f(y)| d y \\
& +\int_{-\infty}^{\bar{a}}|k(x-y)|\left|\chi_{\varepsilon}(x-y)-\chi_{\varepsilon}(a-y)\right||f(y)| d y \\
& =\mathrm{III}+\mathrm{IV} .
\end{aligned}
$$

Observe that $x-y>2(x-a)$ for all $y<\bar{a}$. Then applying the regularity condition (1.3) we can estimate III in the following way:

$$
\begin{aligned}
\mathrm{III} & \leq B_{3} \int_{-\infty}^{\bar{a}} \frac{(x-a)}{(x-y)^{2}}|f(y)| d y \\
& \leq C\left(x_{i}-x_{i+1}\right) \int_{-\infty}^{\bar{a}} \frac{|f(y)|}{(a-y)^{2}} d y \\
& \leq C\left(x_{i}-x_{i+1}\right) \sum_{j=0}^{\infty} \int_{a-(a-\bar{a}) 2^{j+1}}^{a-(a-\bar{a}) 2^{j}} \frac{|f(y)|}{(a-y)^{2}} d y \\
& \leq C\left(x_{i}-x_{i+1}\right) \sum_{j=0}^{\infty} \frac{1}{\left((a-\bar{a}) 2^{j}\right)^{2}} \int_{a-(a-\bar{a}) 2^{j+1}}^{\xi_{0}}|f(y)| d y \\
& \leq \tilde{C} M^{-} f\left(\xi_{0}\right) \leq \tilde{C} \gamma \lambda<\lambda / 4,
\end{aligned}
$$


if $\gamma_{0}<1 /(4 \widetilde{C})$. On the other hand, IV can be estimated applying the size condition on $k$ given by (1.2),

$$
\begin{aligned}
\mathrm{IV} & \leq B \int_{-\infty}^{\bar{a}} \frac{1}{x-y}\left|\chi_{\varepsilon}(x-y)-\chi_{\varepsilon}(a-y)\right||f(y)| d y \\
& =B \int_{(-\infty, \bar{a}) \cap(a-\varepsilon, x-\varepsilon)} \frac{|f(y)|}{x-y} d y .
\end{aligned}
$$

The last term is zero for $\epsilon \leq a-\bar{a}$ since the domain is empty. For $\epsilon>a-\bar{a}$ it is bounded by a constant times $M^{-} f\left(\xi_{0}\right)$. Choosing $\gamma_{0}$ small enough we also have $\mathrm{IV} \leq \lambda / 4$ and the lemma is proved.

Proof of Theorem (2.6). We shall only prove statement (a) since the proof of (b) is analogous and (c) follows from (a) and (b) because $A_{p}^{+} \cap A_{p}^{-}=A_{p}$.

Let $1 \leq p<\infty$ fixed. Assume that there exists a constant $C$ such that the inequality

$$
w\left(\left\{x \in \mathbb{R}:\left|T_{\alpha}^{*} f(x)\right|>\lambda\right\}\right) \leq \frac{C}{\lambda^{p}} \int_{\mathbb{R}}|f(x)|^{p} w(x) d x
$$

holds for every $\lambda>0$, every $\alpha>0$ and all measurable $f$.

We may assume without loss of generality that $k(z)>0$. Since the kernel is continuous in $z$ by (1.3), there exist positive numbers $\delta, z_{1}$ and $z_{2}$ with $z_{1}<z_{2}$ such that

$$
\left[z_{1}, z_{2}\right] \subset\{x: k(x)>\delta\} .
$$

Let $\gamma=z_{1} / z_{2}$ and $\beta=(1-\gamma) / 2$. Let $a<d$ and let us take $b$ and $c$ such that $a<b<c<d$ with $c-b=\gamma(d-a)$ and $d-c=b-a=\beta(d-a)$. Now choose $\alpha=z_{2} /(d-a)=z_{1} /(c-b)$. Then

$$
z_{1}=\alpha(c-b) \leq \alpha(x-y) \leq \alpha(d-a)=z_{2}
$$

for all $x \in(c, d)$ and all $y \in(a, b)$. Therefore, if $x \in(c, d), f=\sigma \chi_{(a, b)}, \sigma=w^{-\frac{1}{p-1}}$, we obtain

$$
\left|T_{\alpha}^{*} f(x)\right| \geq\left|\int_{a}^{b} \sigma(y) k_{\alpha}(x-y) d y\right| \geq \alpha \delta \int_{a}^{b} \sigma(y) d y .
$$

Since the maximal operators $T_{\alpha}^{*}$ are uniformly bounded we get

$$
\int_{c}^{d} w \leq \frac{C}{(\delta \alpha)^{p}}\left(\int_{a}^{b} \sigma\right)^{1-p}=\frac{C(d-a)^{p}}{\delta^{p} z_{2}^{p}}\left(\int_{a}^{b} \sigma\right)^{1-p}
$$

and therefore, keeping in mind that $\delta$ and $z_{2}$ are constants which depend only on the kernel $k$, we have

$$
\int_{c}^{d} w \leq C(d-c)\left(\frac{b-a}{\int_{a}^{b} \sigma}\right)^{p-1} \leq C(d-c)\left(M_{\sigma}\left(\sigma^{-1} \chi_{(a, d)}\right)(a)\right)^{p-1}
$$

where

$$
M_{\sigma} f(x)=\sup _{h, k>0} \frac{\int_{x-h}^{x+k}|f| \sigma}{\int_{x-h}^{x+k} \sigma}
$$


Now, let $x_{0}=d$ and for all nonnegative integer $i$, let $x_{i+1}$ be such that $x_{i}-x_{i+1}=$ $\beta\left(x_{i}-a\right)$. Then inequality $(2.12)$ applied to $a, x_{i+1}, x_{i}$, gives

$$
\begin{aligned}
\int_{x_{i+1}}^{x_{i}} w & \leq C\left(x_{i}-x_{i+1}\right)\left(M_{\sigma}\left(\sigma^{-1} \chi_{\left(a, x_{i}\right)}\right)(a)\right)^{p-1} \\
& \leq C\left(x_{i}-x_{i+1}\right)\left(M_{\sigma}\left(\sigma^{-1} \chi_{(a, d)}\right)(a)\right)^{p-1} .
\end{aligned}
$$

Summing up in $i$ and dividing by $d-a$

$$
\frac{1}{d-a} \int_{a}^{d} w \leq C\left(M_{\sigma}\left(\sigma^{-1} \chi_{(a, d)}\right)(a)\right)^{p-1}
$$

Now, it follows easily from this inequality that

$$
M\left(w \chi_{(a, d)}\right)(a) \leq C\left(M_{\sigma}\left(\sigma^{-1} \chi_{(a, d)}\right)(a)\right)^{p-1},
$$

which is equivalent to $w \in A_{p}^{-}$(see Theorem 2 in $[\mathrm{MPT}]$ for $A_{p}^{-}$weights).

\section{REFERENCES}

[AS] K.F. Andersen and E. T. Sawyer, Weighted norm inequalities for the Riemann-Liouville and Weyl fractional integral operators, Trans. Amer. Math. Soc. 308 (1988), 547-557. MR 89h:26006

[CF] R. Coifman and C. Fefferman, Weighted norm inequalities for maximal functions and singular integrals, Studia Math. 51 (1974), 241-250. MR 50:10670

[HMW] R.A. Hunt, B. Muckenhoupt and R.L. Wheeden, Weighted norm inequalities for the conjugate function and the Hilbert transform, Trans. Amer. Math. Soc. 176 (1973), 261274. MR 47:701

[MOT] F. J. Martín-Reyes, P. Ortega Salvador and A. de la Torre, Weighted inequalities for onesided maximal functions, Trans. Amer. Math. Soc. 319 (1990), 517-534. MR 90j:42047

[MPT] F.J. Martín-Reyes, L. Pick and A. de la Torre, $A_{\infty}^{+}$condition, Canadian Journal of Mathematics 45 (1993), 1231-1244. MR 94m:42042

[MT] F. J. Martín-Reyes and A. de la Torre, Two weight norm inequalities for fractional onesided maximal operators, Proc. Amer. Math. Soc. 117 (1993), 483-489. MR 94b:42010

[S] E. Sawyer, Weighted inequalities for the one sided Hardy-Littlewood maximal functions, Trans. Amer. Math. Soc. 297 (1986), 53-61. MR 87k:42018

[T] A. Torchinsky, Real variable methods in Harmonic Analysis, Academic Press, Inc., 1986. MR 88e: 42001

Dept. Matematica, FiQ, Prop.CAI+D, inteC, Gëmes 3450, 3000 Santa Fe, Argentina

E-mail address: haimar@fiqus.unl.edu.ar

Análisis Matemático, Facultad de Ciencias, Universidad de Málaga, 29071 Málaga, SPAIN

E-mail address: martin_reyes@ccuma.uma.es 